\title{
Tobacco smoking and alcohol consumption as risk factors for glioma: a case-control study in Melbourne, Australia
}

Susan F Hurley, John J McNeil, Geoffrey A Donnan, Andrew Forbes, Michael Salzberg, Graham G Giles

\begin{abstract}
Objective - To investigate possible associations between tobacco smoking and alcohol consumption and the risk of adult glioma.
\end{abstract}

Design - This was a population based, case-control study. Relative risks (RR) were estimated using logistic regression analysis.

Setting - Melbourne, Australia.

Participants - These comprised 416 case subjects (166 women, 250 men), $66 \%$ of those eligible; and 422 control subjects (170 women, 252 men), $43.5 \%$ of those potentially eligible.

Results - There was no increase in risk of glioma with having ever smoked tobacco (RR 1.29, 95\% CI 0.95, 1.75) for all subjects, adjusted for age, a reference date, and gender. There was a slight increase in risk for men (RR 1.64, 95\% CI $1.1,2.45$ ), but not for women (RR 0.99, 95\% CI 0.62, 1.62). For men, there was no increase in risk with increasing packyears of cigarette smoking, but the risk was significantly increased in subjects who had smoked for less than 10 years. There was no increase in risk associated with having ever drunk alcohol for all subjects (RR 0.96, 95\% CI 0.67, 1.37), women (RR $0.69,95 \%$ CI $0.4,1.15$ ) or men (RR 1.40, 95\% CI 0.81, 2.43).

Conclusions - This study does not support an association between either tobacco smoking or alcohol consumption and glioma. The pattern of risk associated with tobacco smoking in men appears inconsistent with a causal role, and may be due to chance, response bias, or uncontrolled confounding.

(F Epidemiol Community Health 1996;50:442-446)

Hypotheses that tobacco smoke and alcohol increase the risk of brain tumours arose because these agents contain $\mathrm{N}$-nitroso compounds, which induce brain tumours in animals. ${ }^{12}$ A number of epidemiological studies have investigated the role of smoking and alcohol consumption as risk factors for brain tumours, ${ }^{3-5}$ but results have been inconclusive. Major difficulties in interpreting previous studies are due to relatively small sample sizes, ${ }^{367}$ inclusion of a mix of histological types, ${ }^{8}$ or the use of hospital control subjects. ${ }^{346}$ The goal of our study was to investigate the role of tobacco smoking and alcohol consumption as risk factors for histologically confirmed glioma in adults in a large, population based, casecontrol study.

\section{Methods}

BACKGROUND

The study was conducted within the Melbourne adult brain tumour study, a casecontrol study designed initially to investigate associations between glioma risk and occupational exposure to chemicals and electromagnetic fields. Information on exposure to other possible risk factors for brain tumour was also collected, using a questionnaire based on an instrument provided by the surveillance of environmental aspects related to cancer in humans (SEARCH) programme of the International Agency for Research on Cancer (IARC). The results of a study of Toxoplasma gondii antibody, conducted in collaboration with another Australian group, have been reported previously.

CASE AND CONTROL SUBJECT SELECTION

Cases were defined as people with a histologically confirmed primary glioma (ICD-0 codes 938-946) diagnosed between July 1987 and December 1991, who were aged 20-70 years at diagnosis, and lived in Melbourne or four other population centres. Residents of these areas account for $85 \%$ of the population of the state of Victoria. Case subjects were identified by screening the medical records of 14 Melbourne hospitals, which together provide almost all the neurosurgical services within Victoria. Completeness of ascertainment was checked by reference to the Victorian cancer registry, to which notification of incident cases of cancer is required by law. We sought approval on an individual basis from the attending neurosurgeon to approach each subject. A letter inviting the patient to participate in an interview was then sent. If the patient did not contact the study office, they were telephoned or a further letter was sent. If the patient had died a proxy subject was interviewed. Proxies were chosen in the following order of priority: spouse (or partner), children, siblings, and other relatives or friends.

Over the study period, we identified and attempted to recruit 627 eligible patients. The attending neurosurgeons refused permission to approach 76 patients, we were unable to contact 67 patients, 15 patients refused to par- 
Table 1 Characteristics of subjects

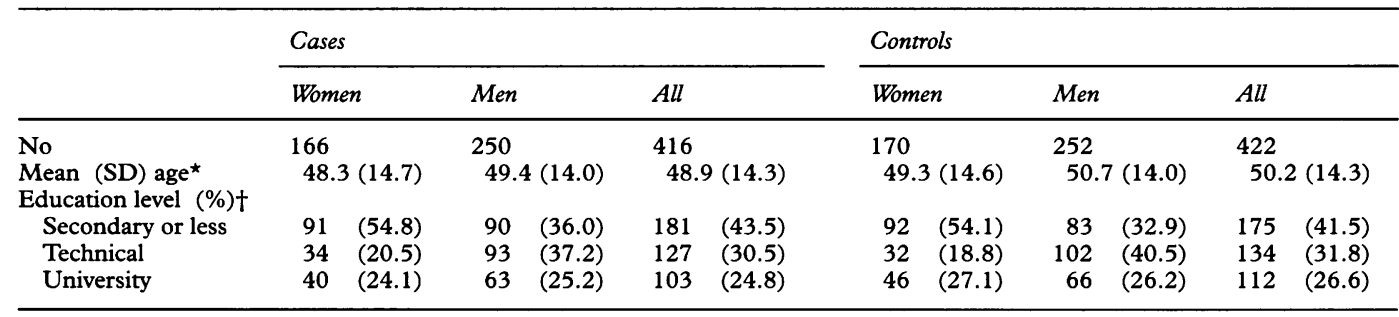

* At reference date (date of diagnosis for cases and selection for controls); † data missing for 5 cases and 1 control.

ticipate, 49 potential proxy subjects refused interview, and four patients did not speak English sufficiently well to be interviewed. We conducted 416 case interviews, $234(56.3 \%)$ with the index subject and $182(43.7 \%)$ with a proxy. Case participation therefore represented $66 \%$ of those eligible and $86 \%$ of those contacted. Of the 416 participating cases, 166 $(39.9 \%)$ were women and $250(60.1 \%)$ were men (see table 1).

A random sample of potential control subjects was selected from the electoral roll, frequency matched to the expected age and gender distribution of cases (predicted from Victorian cancer registry incidence data). Potential controls were considered ineligible if they had a previous diagnosis of brain tumour, stroke, or epilepsy. The latter two exclusions were made because patients with brain tumour can initially present with epilepsy or stroke. Controls were recruited by personal invitation letter, followed by telephone calls or a second invitation letter if the subject did not respond. An index subject was interviewed for each case, irrespective of whether a proxy or index case subject was interviewed.

A total of 968 potential control subjects was selected from the electoral roll. Of these 968, 288 could not be contacted (either because letters were returned to sender or because the subject did not respond to the letter and a telephone number could not be found), 14 had moved interstate or overseas, two were ill, five had died, seven gave a history of stroke, epilepsy or brain tumour, and 230 refused interview. We therefore interviewed 422 control subjects, representing $43.5 \%$ of those identified as potentially eligible and $64.7 \%$ of eligible subjects who were contacted. Of the participating controls, $170(40.3 \%)$ were women and $252(59.7 \%)$ were men.

\section{DATA COLLECTION}

Before the interview, subjects were sent a section of the questionnaire which sought details of passive smoking exposure, household pets, intake of a comprehensive list of foods containing N-nitroso compounds or their precursors, places of residence, and a lifetime occupation list. The main questionnaire was then administered in the subject's home by a research nurse. Details of educational level, smoking history, and alcohol consumption were recorded. Subjects were asked about the amount, frequency, and duration of smoking for tobacco products. Similarly, a lifetime alcohol drinking history was obtained for beer, white and red wine, fortified wine (sherry and port), spirits, and liqueurs. In addition, details of exposure to a large number of other agents which have been suggested as risk factors for brain tumour were sought.

\section{ANALYSIS}

For both smoking and alcohol, the two year period before diagnosis (for cases) and selection for the study (for controls) was excluded when determining exposure status, to allow for a tumour induction period and to avoid consideration of exposures related to early disease. Variables indicating whether the subject had ever smoked any tobacco product and particular types of products were calculated. Only subjects who had smoked at least once a day for three months or more were regarded as having ever smoked. The number of pack-years of cigarette smoking (one pack-year is equivalent to smoking 20 cigarettes per day for one year) over the subject's lifetime was calculated and categorised into three groups based on tertiles of exposure for controls. Variables indicating the age at which the subject started smoking ( $<20$ years or $\geqslant 20$ years), and the duration of smoking $(<10,10-19$, and $\geqslant 20$ years) were also calculated. Exposure to passive smoking was summarised in terms of whether or not the subject had lived, as an adult, with a person who smoked.

Variables were calculated indicating whether the subject had ever drunk any type of alcohol, defined as drinking an alcoholic beverage at least once a month for a year or more, and each type of alcoholic beverage. The number of grams of alcohol consumed over the subject's lifetime was calculated using information on standard drink sizes and the alcohol content of different beverages. ${ }^{10}$ Average daily alcohol consumption over the period they reported drinking was then calculated and classified into three groups based on tertiles of daily alcohol consumption for the control group.

Standard techniques for analysis of casecontrol studies were used. ${ }^{11}$ Logistic regression analysis was used to control for age, gender, and the subject's reference date, and to adjust for any confounding effects. The reference date was the diagnosis date for cases and the selection date for controls, classified into eight categories, each spanning approximately six months. A variable was considered to be a confounder if it altered the risk associated with tobacco smoking or alcohol consumption by $10 \%$ or more. Relative risks were estimated using the method of maximum likelihood, and $95 \%$ confidence intervals (CI) were based on the standard error of coefficient estimates and 
Table 2 Relative risks for tobacco smoking for all subjects, women and men

\begin{tabular}{|c|c|c|c|c|c|c|c|c|}
\hline & \multicolumn{4}{|l|}{ All subjects } & \multicolumn{2}{|c|}{ Women } & \multicolumn{2}{|l|}{ Men } \\
\hline & $\begin{array}{l}\text { No of exposed } \\
\text { cases }\end{array}$ & $\begin{array}{l}\text { No of exposed } \\
\text { controls }\end{array}$ & $R R^{*}$ & $(95 \% C I)$ & $R R t$ & $(95 \% C I)$ & $R R t$ & $(95 \% C I)$ \\
\hline \multicolumn{9}{|c|}{ Smoked any tobacco product $\ddagger$} \\
\hline Never & & & 1.0 & & 1.00 & & 1.00 & \\
\hline Ever & 242 & 232 & 1.29 & $(0.95,1.75)$ & 0.99 & $(0.62,1.62)$ & 1.64 & $(1.10,2.45)$ \\
\hline \multicolumn{9}{|c|}{ Pack-years of cigarette smoking $\oint$} \\
\hline None & & & 1.0 & & 1.00 & & 1.00 & \\
\hline$>0-<9$ & 76 & 72 & 1.19 & $(0.79,1.80)$ & 0.89 & $(0.47,1.70)$ & 1.59 & $(0.91,2.79)$ \\
\hline $9-<24$ & 63 & 81 & 1.01 & $(0.66,1.54)$ & 0.77 & $(0.37,1.61)$ & 1.20 & $(0.71,2.04)$ \\
\hline$\geqslant 24$ & 62 & 77 & 1.04 & $(0.66,1.64)$ & 1.06 & $(0.66,1.71)$ & 1.23 & $(0.71,2.12)$ \\
\hline \multicolumn{9}{|c|}{ Duration of smoking (y) } \\
\hline Never smoked & & & 1.00 & & 1.00 & & 1.00 & \\
\hline$<10$ & 54 & 43 & 1.37 & $(0.84,2.24)$ & 0.75 & $(0.35,1.60)$ & 2.49 & $(1.25,4.92)$ \\
\hline $10-<20$ & 52 & 59 & 1.05 & $(0.66,1.68)$ & 1.10 & $(0.45,2.68)$ & 1.12 & $(0.64,1.97)$ \\
\hline$\geqslant 20$ & 117 & 128 & 1.25 & $(0.86,1.83)$ & 1.17 & $(0.63,2.19)$ & 1.48 & $(0.90,2.42)$ \\
\hline \multicolumn{9}{|c|}{ Age when subject started smoking $(y)^{\star \star}$} \\
\hline Never smoked & & & 1.00 & & 1.00 & & 1.00 & \\
\hline$<20$ & 172 & 170 & 1.21 & $(0.85,1.64)$ & 1.17 & $(0.67,2.08)$ & 1.42 & $(0.93,2.18)$ \\
\hline$\geqslant 20$ & 68 & 62 & 1.48 & $(0.80,1.93)$ & 0.78 & $(0.40,1.52)$ & 2.72 & $(1.48,5.02)$ \\
\hline
\end{tabular}

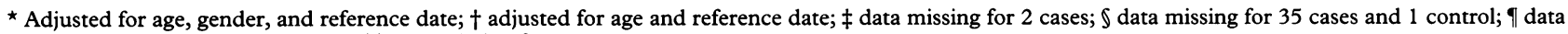
missing for 21 cases and 2 controls; ${ }^{\star \star}$ data missing for 4 cases.

Table 3 Relative risks for alcohol consumption for all subjects, women and men

\begin{tabular}{|c|c|c|c|c|c|c|c|c|}
\hline & \multicolumn{4}{|l|}{ All subjects } & \multicolumn{2}{|c|}{ Women } & \multicolumn{2}{|l|}{ Men } \\
\hline & $\begin{array}{l}\text { No of exposed } \\
\text { cases }\end{array}$ & $\begin{array}{l}\text { No of exposed } \\
\text { controls }\end{array}$ & $R R^{*}$ & $(95 \% C I)$ & $R R t$ & $(95 \% C I)$ & $R R t$ & $(95 \% C I)$ \\
\hline \multicolumn{9}{|c|}{ Drank any alcoholic beveragesł } \\
\hline Never & & & 1.00 & & 1.00 & & 1.00 & \\
\hline Ever & 318 & 325 & 0.96 & $(0.67,1.37)$ & 0.69 & $(0.42,1.15)$ & 1.40 & $(0.81,2.43)$ \\
\hline \multicolumn{9}{|c|}{ Average daily alcohol consumption $\$$} \\
\hline None & & & 1.00 & & 1.00 & & 1.00 & \\
\hline$<10.7 \mathrm{~g} / \mathrm{d}$ & 99 & 108 & 0.79 & $(0.52,1.23)$ & 0.55 & $(0.30,0.99)$ & 1.30 & $(0.66,2.54)$ \\
\hline $10.7-<29.7 \mathrm{~g} / \mathrm{d}$ & 88 & 108 & 0.79 & $(0.51,1.24)$ & 0.67 & $(0.34,1.36)$ & 1.00 & $(0.64,1.89)$ \\
\hline$>29.7 \mathrm{~g} / \mathrm{d}$ & 96 & 106 & 1.02 & $(0.64,1.62)$ & 0.93 & $(0.38,2.25)$ & 1.36 & $(0.73,2.51)$ \\
\hline \multicolumn{9}{|l|}{ Ever drank } \\
\hline Beer & 231 & 244 & 0.90 & $(0.59,1.38)$ & 0.96 & $(0.52,1.79)$ & 0.62 & $(0.31,1.23)$ \\
\hline White wine & 153 & 168 & 0.80 & $(0.57,1.12)$ & 0.61 & $(0.32,1.17)$ & 0.89 & $(0.60,1.35)$ \\
\hline Red wine & 117 & 120 & 0.99 & $(0.70,1.40)$ & 0.71 & $(0.37,1.37)$ & 1.13 & $(0.75,1.71)$ \\
\hline Fortified wine & 98 & 111 & 0.80 & $(0.56,1.14)$ & 1.13 & $(0.59,2.17)$ & 0.68 & $(0.44,1.06)$ \\
\hline Spirits & 138 & 131 & 1.19 & $(0.85,1.68)$ & 0.94 & $(0.51,1.75)$ & 1.35 & $(0.89,2.03)$ \\
\hline
\end{tabular}

* Adjusted for age, gender, and reference date; † adjusted for age and reference date; $¥$ data missing for 1 case; $₫$ data missing for 36 cases and 3 controls; $\uparrow$ risks are relative to subjects who reported drinking alcoholic beverages other than the one indicated.

the normal approximation. Significance tests, at the $5 \%$ level, were performed by referring the likelihood ratio statistic to the $\chi^{2}$ distribution. Where tests for trend were appropriate, the Cochran-Armitage test was used. Most analyses were repeated separately for women and men, controlling for age and reference date. In some instances, analyses were repeated including only index subject interviews (ie excluding proxies). $S A S$ software was used for all computations. ${ }^{12}$

\section{Results}

The risk of glioma, adjusted for age, gender, and reference date, for subjects who had ever smoked a tobacco product relative to those who had never smoked tobacco, was 1.29 (table 2) and the risk for subjects who had ever drunk alcohol, relative to those who had never drunk alcohol, was 0.96 (table 3). Adjustment for education level (table 1) did not substantially alter either risk and it was therefore not regarded as a confounder. The relative risk for ever smoking tobacco, adjusted for ever drinking alcohol was $1.31(95 \% \mathrm{CI} 0.96,1.79)$, and the relative risk for ever drinking alcohol, adjusted for ever smoking tobacco, was 0.89 (95\% CI 0.61, 1.29). As there was little evidence that alcohol confounded any risk associated with smoking, or vice versa, subse- quent analyses focused on smoking and alcohol separately.

SMOKING

As table 2 suggests, the slight elevated risk associated with ever smoking was not statistically significant for all subjects combined, but was marginally statistically significant for men $(p<0.05)$. For women, the relative risk for smoking was close to one. A previously reported association ${ }^{4}$ between smoking nonfilter cigarettes and risk of brain tumour was not confirmed in our analysis. The risk for subjects who had ever smoked non-filter cigarettes relative to those who had smoked tobacco products other than non-filter cigarettes was 1.05 (95\% CI $0.67,1.61)$.

The results of analyses investigating the effects of pack-years of cigarette smoking, duration of smoking and age when the subject started smoking are presented in table 2 . For men, there was no evidence of a trend in risk of glioma with increasing pack-years of cigarette smoking. The highest relative risk (1.59) was for the lowest category of pack-years of exposure. There was a statistically significant difference $(p<0.025)$ in risk with duration of smoking, with men who had smoked for fewer than 10 years having the highest risk. Smoking duration and age at the start of smok- 
ing are highly correlated, and, as would be expected given the results for duration of smoking, there was a significant difference in risk with age at the start of smoking ( $p=$ 0.005). Men who started smoking later (after age 20 years) had the highest risk: 2.72 relative to men who never smoked. There was no statistically significant modification according to age at the reference date (classified as $<40$ years, $40-59$ years, $\geqslant 60$ years) of the effects of duration of smoking or age when the subject started smoking. Similar, but weaker, patterns for the three indicators of smoking exposure considered were seen for all subjects. For women, relative risks were all close to one (table 2).

Our finding of a higher risk of glioma for men who had started smoking at an older age, and hence had smoked for shorter periods than men of the same age, is difficult to reconcile with a role for tobacco smoking in the aetiology of glioma. Examination of the regression model diagnostics did not suggest that this result was due to an outlying value. We therefore investigated the possibility that the lag period of two years was too short, and that the increased risk associated with more recent commencement of smoking reflected a change in habits as the brain tumour developed, perhaps to alleviate headaches. The analysis was repeated excluding exposure in the 10 years before diagnosis (for cases) or selection (for controls). Similar results were obtained, with a risk of $2.48(95 \%$ CI $1.35,4.57 ; 44$ cases and 27 controls) for men who started smoking at age 20 years or later, and a risk of 1.83 (95\% CI $0.90,3.72 ; 26$ cases and 18 controls) for men who had smoked for less than 10 years (both relative to non-smokers).

Results were also similar when data from interviews with 182 proxies for case subjects were excluded. The relative risks associated with having ever smoked were increased slightly to 1.51 (95\% CI $1.05,2.17)$ for all subjects, $1.23(95 \%$ CI $0.68,2.20)$ for women, and 1.73 (95\% CI 1.07, 2.78) for men. The patterns described above for the other measures of smoking exposure were also found for non-proxy interviews.

\section{PASSIVE SMOKING}

The effect of passive smoking was investigated in two ways. First, in line with the recommendation of Wells, ${ }^{13}$ the risk associated with smoking was recalculated relative to the non-smokers (96 cases and 109 controls) who had not been exposed to ambient tobacco smoke at home. There was little change in risks for having ever smoked using this reference group: relative risks were 1.32 (95\% CI 0.92 , 1.89) for all subjects, 0.89 (95\% CI 0.48 , $1.61)$ for women, and 1.78 (95\% CI 1.11 , 2.84) for men. Second, the effect of passive smoking was investigated in the 172 cases and 190 controls who had never smoked themselves. The risk of glioma for those who had lived with a smoker ( 76 cases and 81 controls) relative to those who had not was 0.97 (95\% CI 0.61, 1.53).
ALCOHOL

As mentioned above, there was no evidence of an association between ever drinking alcohol and glioma for all subjects. The relative risk associated with having ever drunk alcohol was decreased for women and increased for men, but neither differed significantly from one (table 3). There was no statistically significant difference in risk for different levels of alcohol consumption. Furthermore, none of the relative risks for drinking particular types of alcohol differed significantly from one, although for both women and men there was a non-significant decrease in risk associated with drinking white wine (table 3).

Results were similar when proxy interviews were excluded. The relative risks associated with having ever drunk alcohol were 0.91 (95\% CI $0.59,1.39)$ for all subjects, $0.72(95 \% \mathrm{CI}$ $0.39,1.32)$ for women and $1.13(95 \%$ CI 0.42 , 3.04) for men.

\section{Discussion}

Nine previous case-control studies have investigated tobacco smoking as a risk factor for glioma. An exploratory study by Burch et $a l^{4}{ }^{4}$ using hospital control subjects, reported a relative risk of 1.44 (95\% CI $0.94,2.21)$ for having ever smoked non-filter cigarettes, and a significant trend in risk with increased use of this product. Eight other studies found no association between tobacco use and brain tumours. ${ }^{3-8}{ }^{14-16}$ All of these studies had shortcomings. The maximum number of cases studied was only $215,{ }^{4}$ and three studies included fewer than 100 cases of glioma. ${ }^{37}$ In one study, results were reported for glioma and meningioma combined, ${ }^{8}$ although the aetiology of these two tumours is likely to differ. Hospital controls were used in three studies, ${ }^{346}$ and people with other cancers in one study. ${ }^{15}$ Information on smoking history was obtained from medical records in one study, ${ }^{7}$ and cancer registry data in another. ${ }^{15}$

In our analysis, we found an increase, of marginal statistical significance, in the risk of glioma for men who had smoked tobacco. However, the pattern of risk associated with duration of smoking appears inconsistent with a causal role. Despite the limitations of previous studies, and our own, the results taken together do not support the hypothesis that tobacco smoking is a risk factor for glioma.

Our finding of an increased risk of glioma for men who had smoked tobacco, particularly those who had smoked for short periods of time, could be due to chance, response bias, or uncontrolled confounding. The possibility of response bias cannot be excluded, particularly in view of the suboptimal participation rates, especially for control subjects. However, the participation rate in our study for control subjects who could be contacted was comparable with response rates for the two previous studies which were population based. ${ }^{816}$ Another potential source of response bias was use of proxy respondents for patients who had died, but index subjects for all controls. ${ }^{17}$ However, our conclusions were unaltered when analyses were restricted to index subjects, suggesting 
that any bias introduced by this aspect of study design was minimal for these exposures. Control of confounding was difficult because of the lack of proven risk factors for glioma, and confounding due to occupational or other exposures may explain the observed association with tobacco smoking in men.

Our investigation of the role of passive smoking was motivated by Hirayama's cohort study of 91540 non-smoking Japanese women, which found an increased brain tumour mortality rate for women with husbands who were smokers. ${ }^{18}$ The analysis was based on only 34 cases. An association between passive smoking and glioma risk, in the absence of an association for active smoking, would be surprising, and we did not find such an association. However, Ryan et al did report an increased risk of meningioma for passive smoking, but no association for active smoking. ${ }^{5}$

We found no evidence of an association between having ever drunk alcohol and glioma, but did find a decrease in risk, which was not statistically significant, for both women and men who reported drinking white wine. Six of the nine case-control studies mentioned above also investigated the role of alcohol as a risk factor for brain tumour. Four studies ${ }^{36716}$ found no association, the study by Burch et al ${ }^{4}$ found an increased risk for drinkers of wine, and Ryan $e t a t$ found a decreased risk of glioma (and meningioma) for drinkers of white wine. Therefore, the results of previous studies and our own do not support the hypothesis that drinking alcohol increases the risk of glioma. The reduction in risk associated with white wine reported by Ryan et $a l,{ }^{5}$ and seen to some extent in our data, may warrant further investigation, but we are unaware of a possible mechanism for a protective effect of drinking white wine.

In summary, our study does not support associations between tobacco smoking or alcohol consumption and the risk of glioma. When our results and those of previous studies are considered together it seems unlikely that either smoking or alcohol are risk factors for glioma.
We thank all the subjects who participated; the nurseinterviewers, Judy Snaddon and Sarah Gardiner; Lichun Quang for computer programming assistance; and Ieva Ozolins for research assistance. We would particularly like to thank the participating neurosurgeons whose collaboration made this sur possible. The study was funded by grants from the this study possible. The study was funded by grants from the Nationa Health and Medical Research Council, the Victorian Health Promotion Foundation, and the Alfred Hospital. Susan Hurley is supported by a Neil Hamilton Fairley Fellowship (Number 927215) from the National Health and Medical Research Council.

1 Swenberg JA. Chemical- and virus-induced brain tumors. Natl Cancer Inst Monogr 1977;46:3-10.

2 Rubinstein LJ. Correlation of animal brain tumor models with human neuro-oncology. Natl Cancer Inst Monogr 1977;46:43-9.

3 Ahlbom A, Navier IL, Norell S, Olin R, Spõnnare B. Nonoccupational risk indicators for astrocytomas in adults. $A m$ Ocpidemiol 1986;124:334-7.

4 Burch JD, Craib KJP, Choi BCK, Miller AB, Risch HA Howe GR. An exploratory case-control study of brain tumors in adults. $\mathcal{F}$ Natl Cancer Inst 1987;78(4):601-9.

5 Ryan P, Lee MW, North B, McMichael AJ. Risk factors for tumours of the brain and meninges: results from the Adelaide adult brain tumor study. Int $f$ Cancer 1992; 51:20-7.

6 Musicco M, Filippini G, Bordo BM, Melotto A, Morello G, Berrino F. Gliomas and occupational exposure to carcinogens: case-control study. Am $\mathcal{F}$ Epidemiol 1982;116: $782-90$

7 Carpenter AV, Flanders WD, Frome EL, Cole P, Fry SA. Brain cancer and nonoccupational risk factors: a casecontrol study among workers at two nuclear facilities. $A m$ 7 Public Health 1987;77:1180-2.

8 Schlehofer B, Kunze S, Sachsenheimer W, Blettner M, Niehoff $\mathrm{D}$, Wahrendorf J. Occupational risk factors for brain tumors: results from a population-based case-contro study in Germany. Cancer Causes Control 1990;1:209-15.

9 Ryan P, Hurley SF, Johnson AM, et al. Tumours of the brain and presence of antibodies to Toxoplasma gondii. Int $\mathcal{F}$ Epidemiol 1993;22:412-19.

10 Commonwealth Department of Health. Metric tables of com position of Australian foods. Canberra: Commonwealth position of Australian foods.

11 Breslow NE, Day NE. Statistical methods in cancer research Vol 1: Case-control studies. Lyon: IARC, 1980

12 SAS Institute. SAS Technical Report P-200 SAS/STAT Software: CALIS and LOGISTIC Procedures, Release 6.04. Cary, NC: SAS Institute Inc, 1990.

13 Wells JA. Breast cancer, cigarette smoking, and passive smoking ?. Am $\mathcal{F}$ Epidemiol 1991;133:208-10.

14 Hochberg F, Toniolo P, Cole P. Nonoccupational risk indicators of glioblastoma in adults. $\mathcal{7}$ Neurooncol 1990;8:5560 .

15 Brownson RC, Reif JS, Chang JC, Davis JR. An analysis of occupational risks for brain cancer. Am $\mathcal{f}$ Public Health 1990;80:169-72.

16 Preston-Martin S, Mack W, Henderson BE. Risk factors fo gliomas and meningiomas in males in Los Angeles County. Cancer Res 1989;49:6137-43.

17 Nelson LM, Longstreth WTi, Koepsell TD, van Belle G. Proxy respondents in epidemiologic research. Epidemiol Rev 1990;12:71-86.

18 Hirayama T. Cancer mortality in nonsmoking women with smoking husbands based on a large-scale cohort study in Japan. Prev Med 1984;13:680-90. 